\title{
A PRODUÇÃO ACADÊMICA INTERNACIONAL EM PRÁTICAS E INICIATIVAS NA GESTÃO COLABORATIVA EM CADEIAS DE SUPRIMENTOS: UM ESTUDO BIBLIOMÉTRICO
}

\section{THE INTERNATIONAL ACADEMIC PRODUCTION IN PRACTICES AND INITIATIVES OF SUPPLY CHAIN COLLABORATIVE MANAGEMENT: A BIBLIOMETRIC STUDY}

\author{
Valdir Antonio Vitorino Filho* E-mail: valdir.vitorino@gmail.com \\ João Batista de Camargo Júnior** E-mail:ibcir@uol.com.br \\ Silvio Roberto Ignácio Pires** E-mail: sripires@unimep.br \\ Ana Rita Tiradentes Terra Argoud** E-mail: arargoud@unimep.br \\ *Instituto Federal de São Paulo (IFSP), São Paulo, SP \\ **Universidade Metodista de Piracicaba (UNIMEP), Piracicaba, SP
}

\begin{abstract}
Resumo: Este artigo tem como objetivo analisar a produção acadêmica internacional sobre as principais práticas e iniciativas na gestão colaborativa em cadeias de suprimentos em artigos publicados em periódicos internacionais indexados no Scopus. A pesquisa avaliou publicações no período de 2005 a 2014, utilizando-se de técnicas bibliométricas para identificar ano de publicação, país e periódicos em que esses artigos foram publicados e predominância de temas. No total, a pesquisa investigou 331 artigos com as seguintes práticas e iniciativas colaborativas: Electronic Data Interchange (EDI), Vendor Managed Inventory (VMI), Continuous Replenishment (CR), Efficient Customer Response (ECR) e Collaborative Planning, Forecasting and Replenishment (CPFR). Os resultados da pesquisa demonstram que (a) não existe concentração de publicações em poucos periódicos; (b) existe concentração de publicação sobre os temas em poucos países, destacando-se os Estados Unidos; (c) não existe uma concentração de autores com vários artigos publicados sobre alguma das práticas ou iniciativas; (d) o EDI e VMI são as práticas mais investigadas nos artigos analisados e (e) não há concentração das publicações em algum ano específico, mantendo-se equilibradas as publicações durante o período investigado pela pesquisa.
\end{abstract}

Palavras-chave: Gestão colaborativa em cadeia de suprimentos. Práticas e iniciativas colaborativas em cadeias de suprimentos. Bibliometria.

Abstract: This paper aims to analyze the international academic literature production about the main practices and initiatives related to collaborative management in supply chains in articles published in international journals indexed in Scopus. The research evaluated publications in the 2005-2014 period, using bibliometric techniques to identify the publication year, the country and the periodicals in which these papers were published, as well as the predominant themes. In total the research investigated 331 papers related to the following practices and collaborative initiatives: Electronic Data Interchange (EDI), Vendor Managed Inventory (VMI), Continuous Replenishment (CR), Efficient Customer Response (ECR) and Collaborative Planning, Forecasting and Replenishment (CPFR). The results demonstrated that (a) there is no concentration of publications in a small group of journals; (b) there is a concentration of publication about the topics in a few countries, especially the United States; (c) there is no concentration of authors with several articles published about one of the practices or initiatives; (d) the EDI and the VMI are the most investigated practices in the articles analyzed; and (E) there is no concentration of publications in any specific year, keeping the publications balanced over the period investigated by the research.

Revista Produção Online. Florianópolis, SC, v.17, n. 2, p. 567-591, 2017. 
Keywords: Collaborative management in supply chains. Collaborative practices and initiatives in supply chains. Bibliometrics.

\section{INTRODUÇÃO}

O aumento da concorrência e a globalização dos mercados tem impulsionado as empresas a buscar o desenvolvimento tecnológico e uma ampla oferta de produtos e serviços para atender as necessidades de seus clientes. A colaboração entre empresas (concorrentes, fornecedores e distribuidores) tem sido uma alternativa para que as empresas e suas cadeias de suprimentos (supply chain SC) se mantenham competitivas no mercado (CHRISTOPHER e TOWILL, 2001).

Dessa forma, as SC que alcançarem uma estruturação, coordenação e gerência das atividades realizadas com seus parceiros podem obter melhores retornos em relação às SC concorrentes (COX, 2004; VIVALDINI e SOUZA, 2006).

Neste sentido, a gestão colaborativa em SC pode ser compreendida como um conjunto de ações entre duas ou mais empresas que planejam e executam operações de maneira mais ágil e eficaz em comparação ao que realizariam isoladamente (COX, 2004).

A fim de atingir esses objetivos, os elos da cadeia podem utilizar práticas e iniciativas que proporcionem a troca rápida de informações e a visibilidade das operações conjuntas. Entre as principais práticas e iniciativas a serviço do planejamento e gestão colaborativa na SC estão: Electronic Data Interchange (EDI), Vendor Managed Inventory (VMI), Continuous Replenishment (CR), Efficient Customer Response (ECR) e Collaborative Planning, Forecasting and Replenishment (CPFR) (PIRES, 2009). É importante ressaltar que, dessas práticas e iniciativas, o EDI é basicamente uma tecnologia da informação e comunicação (TIC), enquanto as demais correspondem a processos de negócios potencializados e/ou viabilizados pelo uso da tecnologia.

Desse modo, o objetivo desta pesquisa é analisar a produção acadêmica internacional envolvendo o tema "práticas e iniciativas colaborativas em cadeias de suprimentos", delimitando como escopo as principais práticas e iniciativas para o planejamento e gestão colaborativa na SC (EDI, VMI, CR, ECR e CPFR) nos últimos dez anos. Nesse sentido, o estudo pretende dar um panorama a respeito dos temas, Revista Produção Online. Florianópolis, SC, v.17, n. 2, p. 567-591, 2017. 
principalmente ao demonstrar onde, por quem e quais temáticas têm sido mais pesquisadas. A pesquisa utiliza uma amostragem de 331 artigos que se encontram na base do Scopus, publicados no período de 2005 a 2014.

Partindo-se de questões ligadas às referências bibliográficas utilizadas nos estudos das práticas e iniciativas colaborativas em SC, tem-se como questionamento principal: "como se apresenta a pesquisa acadêmica internacional sobre as principais práticas e iniciativas colaborativas em cadeias de suprimentos nos últimos dez anos?".

A partir da elaboração do objetivo e questão de pesquisa deste estudo, foram delimitadas as seguintes proposições: (a) há concentração de publicações em poucos periódicos? (b) existe predominância de publicações em alguns países? (c) há claramente alguns autores com maior número de publicações sobre os temas? (d) existem práticas e iniciativas que se destacam em relação à quantidade de pesquisas? (e) as publicações sobre os temas cresceram conforme o passar dos anos?

Nos últimos anos, têm sido divulgadas diversas pesquisas bibliométricas Knoff et al. (2014), por exemplo, realizaram um mapeamento das publicações científicas sobre estratégia de manufatura, com a utilização do processo ProKnow-C, destacando o periódico Accounting, Organizations and Society na área. Vitorino Filho et al. (2015) desenvolveram um estudo bibliométrico sobre a produção acadêmica internacional em gestão de operações, e os resultados apontam que não existe concentração de autores com várias publicações sobre o tema, que a maioria das publicações tem origem nos Estados Unidos, e que os anos de 2007 e 2010 concentraram um maior número de publicações. Já Rodrigues, Providello e Bagno (2016) analisaram as operações de atendimento à saúde como contexto de aplicação de técnicas de engenharia de produção, com um levantamento na base de dados do Scopus, nos anos de 2004 a 2014, elencando diversas categorias de análises, como autores, países, instituições e ano de publicação.

Nesse sentido, o artigo está estruturado da seguinte forma: na Seção 2, são apresentadas as definições e discussões acerca das principais práticas e iniciativas colaborativas em gestão da cadeia de suprimentos; na Seção 3, apresentam-se a abordagem metodológica e os métodos utilizados na pesquisa; na Seção 4, exibem- 
se os resultados da pesquisa; na Seção 5, tecem-se as considerações finais sobre o estudo; e, por fim, são apresentadas as referências utilizadas para elaboração desta pesquisa.

\section{PRÁtICAS E INICIATIVAS NA GESTÃO COLABORATIVA EM CADEIAS DE SUPRIMENTOS}

Pires (2009) destaca como principais práticas e iniciativas em gestão colaborativa em SC: EDI, VMI, CR, ECR e CPFR - conforme Figura 1. A sequência estabelecida pelo autor tem como base mais os termos de nível de colaboração do que termos cronológicos.

Figura 1 - Evolução das principais práticas e iniciativas em gestão colaborativa

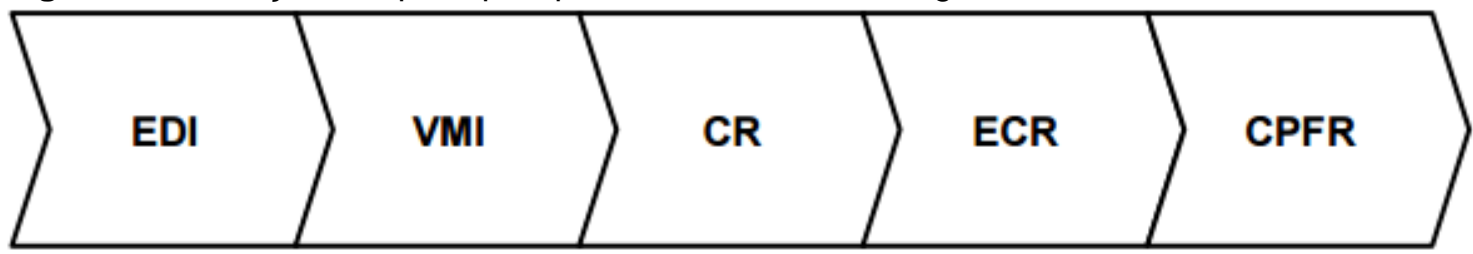

Fonte: Adaptado de Pires (2009, p. 174)

No Quadro 1, destacam-se as principais ferramentas, uma breve contextualização e algumas pesquisas que investigaram essas ferramentas.

Quadro 1 - Ferramentas de gestão colaborativa na cadeia de suprimentos

\begin{tabular}{|c|c|c|}
\hline $\begin{array}{l}\text { Ferramentas de gestão } \\
\text { colaborativa }\end{array}$ & Contextualização & Algumas pesquisas \\
\hline $\begin{array}{l}\text { Electronic Data Interchange } \\
\text { (EDI) }\end{array}$ & $\begin{array}{l}\text { Operações mais coordenadas } \\
\text { entre fornecedores e clientes } \\
\text { através de trocas eletrônicas de } \\
\text { dados via computadores de } \\
\text { empresas que se relacionam } \\
\text { na SC. }\end{array}$ & $\begin{array}{l}\text { Ângelo e Siqueira (2000); } \\
\text { Arunachalam (2004); Machuca e } \\
\text { Barajas (2004); Ferreira e Alves } \\
\text { (2005); Tan, Kannan e Hsu (2010); } \\
\text { Portal EDI (2013). }\end{array}$ \\
\hline $\begin{array}{l}\text { Vendor Managed Inventory } \\
\text { (VMI) }\end{array}$ & $\begin{array}{l}\text { O fornecedor gerencia } 0 \\
\text { estoque do seu cliente. }\end{array}$ & $\begin{array}{l}\text { Blatherwick (1998); Barratt e Oliveira } \\
\text { (2001); Saab Jr. e Corrêa (2008); } \\
\text { Lee e Ren (2011); Yu, Wang e Liang } \\
\text { (2012); Chen (2013); Govindan } \\
\text { (2013); Hariga et al. (2013); Pereira, } \\
\text { Pires e Silva (2013). }\end{array}$ \\
\hline $\begin{array}{l}\text { Continuous Replenishment } \\
\text { (CR) }\end{array}$ & $\begin{array}{l}\text { O controle de estoques baseia- } \\
\text { se na previsão de vendas e na } \\
\text { demanda histórica. }\end{array}$ & $\begin{array}{l}\text { Cachon e Fisher (1997); Wanke } \\
\text { (2004). }\end{array}$ \\
\hline $\begin{array}{l}\text { Efficient Consumer Response } \\
\text { (ECR) }\end{array}$ & $\begin{array}{l}\text { A demanda ou reposição de } \\
\text { itens é puxada pelo ponto de } \\
\text { consumo e baseia-se no ponto } \\
\text { de reposição do estoque just in } \\
\text { time, com foco na reposição } \\
\text { eficiente e gestão de }\end{array}$ & $\begin{array}{l}\text { Ângelo e Siqueira (2000); Wanke } \\
\text { (2001); ECR Brasil (2001); Hoffman e } \\
\text { Mehra (2000); Corsten e Kumar } \\
\text { (2005); Martens e Dooley (2010). }\end{array}$ \\
\hline
\end{tabular}

Revista Produção Online. Florianópolis, SC, v.17, n. 2, p. 567-591, 2017. 


\begin{tabular}{|l|l|l|}
\hline & categorias. & \\
\hline & Prioriza o controle de estoque & Voluntary Interindustry Commerce \\
Collaborative Planning, & em todos os elos da SC com o & Mendards (2004); Resende e \\
Forecasting and & intuito de auxiliar as empresas & Porto (2008); Vivaldini, Pires e Souza \\
Replenishment (CPFR) & a administrar e compartilhar & (2008); Vivaldini, Pires e Souza \\
& informações. & (2010). \\
\hline
\end{tabular}

Fonte: Adaptado de Vitorino Filho et al. (2016)

Além das ferramentas citadas no Quadro 1, outras práticas e iniciativas em SC também são relevantes para o alcance dos objetivos na gestão colaborativa, como relatam os autores Wanke (2004), Rodrigues e Sellitto (2008) e Almeida, Marins e Tramarico (2012): desenvolvimento de fornecedores, outsourcing, in plant representatives (os representantes de fornecedores estão presentes na planta dos clientes) e o Early Supplier Involvement (ESI), que corresponde ao envolvimento dos fornecedores desde a fase inicial do projeto do produto. Nota-se, porém, que essas demais práticas são viáveis em processos específicos, e as comunicações entre as partes se referem a atributos mais simples do que as outras. Desse modo, as principais ferramentas da gestão colaborativa abordadas no Quadro 1 demandam a troca de informações mais frequentes para proporcionar a colaboração necessária entre os envolvidos e incorrem em custos elevados e integração complexa (CAMARGO JUNIOR, 2015). Por esse motivo, é importante detalhar um pouco mais suas características.

\subsection{Electronic Data Interchange}

O EDI, que em português significa Intercâmbio Eletrônico de Dados, surgiu nos Estados Unidos por volta da década de 1940. Porém, apenas na década de 1980 essa tecnologia passou a ser utilizada mais frequentemente por empresas. Desde o princípio, o objetivo básico era a troca eletrônica de dados entre as empresas parceiras, dados esses que poderiam variar desde programas de produção até a emissão de faturas para pagamento (PIRES, 2009).

De acordo com o Portal EDI (2013), essa tecnologia representa um sistema de envio e recebimento de documentos eletrônicos que são compartilhados entre empresas parceiras a partir de uma padronização preestabelecida. Através de sua utilização, proporciona-se o envio rápido dos documentos aos seus respectivos 
destinatários e, além da redução de tempo, as empresas diminuem custos nos processos comerciais e logísticos.

Analisando as possíveis influências da comunicação eletrônica via EDI em relação a algumas atividades operacionais como a gestão de estoques, o transporte e o processamento de pedidos, é possível notar o potencial do EDI, como demonstra o Quadro 2.

Quadro 2 - Influências do EDI

\begin{tabular}{|c|l|}
\hline Atividade & \multicolumn{1}{|c|}{ Influência do EDI } \\
\hline \multirow{3}{*}{ Gestão de estoques } & - A informação com menos erros e em tempo \\
real pode resultar na redução dos níveis de \\
estoques, evitando, assim, custos \\
desnecessários, perda de capital de giro e a \\
obsolescência de produtos; \\
- A diminuição de incertezas e a melhor \\
percepção da demanda proporcionadas pela \\
troca eletrônica de informações permitem \\
maior visibilidade para planejamento e \\
controle de estoques.
\end{tabular}

Fonte: Adaptado de Ferreira e Alves (2005, p. 440)

Assim, Pires (2009) apresenta algumas vantagens e desvantagens do EDI, como segue no Quadro 3.

Quadro 3 - Vantagens e desvantagens do EDI

\begin{tabular}{|l|l|}
\hline \multicolumn{1}{|c|}{ Vantagens } & \multicolumn{1}{c|}{ Desvantagens } \\
\hline $\begin{array}{l}\text { Melhor comunicação e precisão dos dados } \\
\text { transacionados. }\end{array}$ & $\begin{array}{l}\text { Necessidade de padronização dos } \\
\text { documentos. }\end{array}$ \\
\hline Maior rapidez no acesso à informação. & Custo relativamente alto de implementação. \\
\hline Maior produtividade nas transações de dados. & $\begin{array}{l}\text { Necessário uso de softwares e hardwares } \\
\text { padronizados. }\end{array}$ \\
\hline Diminuição dos custos administrativos e de & Necessária a conscientização e padronização \\
\hline
\end{tabular}

Revista Produção Online. Florianópolis, SC, v.17, n. 2, p. 567-591, 2017. 


\begin{tabular}{|l|l|}
\hline transações. & de todos os usuários. \\
\hline Redução de lead times. & Flexibilidade relativamente baixa do sistema. \\
\hline Redução dos estoques. & Dependência dos provedores de serviço. \\
\hline Maior agilidade nas tomadas de decisão. & $\begin{array}{l}\text { Dependência da qualidade dos sistemas de } \\
\text { comunicação utilizados. }\end{array}$ \\
\hline
\end{tabular}

Fonte: Adaptado de Pires (2009, p. 157)

Desse modo, fica claro que o EDI veio para revolucionar a forma como as empresas conduzem suas atividades comerciais (ARUNACHALAM, 2004). O alinhamento relacional entre os parceiros da SC, somado à melhora na comunicação, proporciona ganhos significativos aos elos da cadeia de suprimentos e, por consequência, ao cliente final (TAN, KANNAN e HSU, 2010).

\subsection{Vendor Managed Inventory}

De acordo com Blatherwick (1998), o VMI (em português, Estoque Gerenciado pelo Fornecedor) ganhou força na década de 1990, nos Estados Unidos, com sua aplicação em grandes redes de varejo. Segundo Pires (2009), o VMI pode ser considerado uma prática em que a empresa produtora não mais gerencia seu estoque, e sim seu fornecedor, com sua implantação exigindo um alto nível de integração nas informações, processos e operações entre as empresas envolvidas na SC.

Barratt e Oliveira (2001) defendem que o VMl é uma técnica na qual o produtor tem um único fornecedor responsável pelo gerenciamento de sua política de estoques, e isso inclui o processo de reposição.

Já para Govindan (2013), a prática representa uma metodologia pela qual a parte montante de uma cadeia de suprimentos assume a responsabilidade pela gestão de estoques na parte jusante da cadeia com base em limites anteriormente acordados. O VMI é, assim, uma das primeiras ligações de negócios com base na confiança e troca de informações entre fornecedores e varejistas.

Desse modo, para Lee e Ren (2011), por tratar-se de uma estratégia de parceria que permite a um fornecedor realizar pedidos em nome de seus clientes (HARIGA et al., 2013), exigindo elevado grau de compartilhamento de objetivos, o VMI é uma prática que evidencia a necessidade e importância da colaboração na SC.

Revista Produção Online. Florianópolis, SC, v.17, n. 2, p. 567-591, 2017. 
Nesse sentido, Saab Jr. e Corrêa (2008) descrevem um estudo de caso de insucesso na aplicação do VMl em uma cadeia de suprimentos do setor de bens de consumo não duráveis e concluem que o VMI pode ser subutilizado em termos de informação e em alguns aspectos conceituais importantes quando implantado em cadeias complexas.

Em um compêndio dessas barreiras e oportunidades, Pires (2009) aponta algumas vantagens e desvantagens que ocorrem na implantação do VMI, levando em consideração tanto a empresa fornecedora como a empresa-cliente, conforme apresentado no Quadro 4.

Quadro 4 - Vantagens e desvantagens do VMI

\begin{tabular}{|c|c|c|}
\hline & Empresa fornecedora & Empresa-cliente \\
\hline Vantagens & $\begin{array}{l}\text { Melhor atendimento e maior } \\
\text { fidelização do cliente. } \\
\text { Melhor gestão da demanda. } \\
\text { Melhor conhecimento do } \\
\text { mercado. }\end{array}$ & $\begin{array}{l}\text { Menor custo de estoques e } \\
\text { de capital de giro. } \\
\text { Melhor atendimento por parte } \\
\text { do fornecedor. } \\
\text { Simplificação da gestão dos } \\
\text { estoques e de compras. }\end{array}$ \\
\hline Desvantagens & $\begin{array}{l}\text { Custo do estoque mantido no } \\
\text { cliente. } \\
\text { Custo da gestão do sistema. }\end{array}$ & $\begin{array}{l}\text { Maior dependência do } \\
\text { fornecedor. } \\
\text { Perda do controle sobre seu } \\
\text { abastecimento. }\end{array}$ \\
\hline
\end{tabular}

Fonte: Adaptado de Pires (2009, p. 162)

É importante notar que, mesmo com os custos de inventários transferidos do varejista, os fornecedores podem se beneficiar com o VMI devido às economias de escala na produção e entrega. Assim, os custos totais da cadeia de suprimentos tendem sempre a diminuir com a utilização do VMI, sendo que essa redução é maior quando ocorrem incertezas nas taxas de câmbio, comparando-se a casos em que não há nenhuma incerteza cambial (LEE e REN, 2011).

Yu, Wang e Liang (2012) desenvolveram um estudo sobre a deterioração das matérias-primas e produtos finais envolvidos em uma SC de alimentos e como a aplicação do VMI pôde diminuir as taxas de deterioração desses produtos. $\mathrm{O}$ estudo utilizou um comparativo numérico de custos, que aponta que a taxa de deterioração de um produto pode aumentar os custos totais em mais de $40 \%$ comparando-se a uma taxa de deterioração zero.

Já em um estudo sobre a aplicação do VMI em um hospital público para a gestão de oxigênio líquido e outros materiais de consumo, os resultados demonstram que, apesar da aversão das empresas públicas a aplicações de Revista Produção Online. Florianópolis, SC, v.17, n. 2, p. 567-591, 2017. 
técnicas mais comumente utilizadas por empresas privadas, o VMI mostrou-se uma ferramenta precisa de controle, podendo ser estendida a outros tipos de materiais e a outros setores públicos (PEREIRA, PIRES e SILVA, 2013).

\subsection{Continuous Replenishment}

A Reposição Contínua (tradução para 0 português de Continuous Replenishment) também surgiu na década de 1990 e está intimamente ligada ao processo de gestão por categorias, controlando o estoque e demanda de cada categoria e agindo para sua reposição quando necessário (KURNIA, SWATMAN e SCHAUDER, 1998). Portanto, o processo de CR facilita o gerenciamento de estoques pelos compradores, proporcionando a eles maior tempo para atividades de aumento de vendas e melhoria de rentabilidade.

Para Cachon e Fisher (1997), no CR os varejistas transmitem, via EDI, informações diárias aos fornecedores, que acabam assumindo a responsabilidade da gestão dos estoques de seus clientes.

Segundo Wanke (2004), a estratégia básica de CR consiste em que os fornecedores recebam os dados do PDV (Ponto de Venda) para assim prepararem carregamentos em intervalos regulares que possam garantir a flutuação do estoque do cliente entre determinados níveis máximo e mínimo, visto que a causa dessas variações são os padrões sazonais de demanda, de promoções e algum tipo de mudança nas preferências dos consumidores.

Vivaldini (2007) complementa afirmando que a CR pode ser entendida como a reposição de estoques com base nas vendas e consumo real dos produtos, diferenciando-se do VMI pela maior quantidade no estoque do cliente e levando em consideração a previsão de vendas e a demanda histórica para estabelecimento das políticas de estoques, e não somente as modificações no nível de estoque dos PDV.

Nesse subitem da pesquisa cabe ressaltar que poucos estudos foram encontrados, na literatura nacional e internacional, que tratam especificamente sobre a análise da aplicação do $\mathrm{CR}$, visto que ela acaba aparecendo em conjunto com as demais práticas de colaboração em cadeia de suprimentos. Isso ocorre porque, 
conforme afirmam Kurnia, Swatman e Schauder (1998) e Wanke (2004), a prática pode ser considerada um subprocesso do ECR.

\subsection{Efficient Consumer Response}

Para Pires (2009), a aplicação da tecnologia de informação nos processos da cadeia de suprimentos tem contribuído para a redução do nível de burocracia e na diminuição dos custos de transação.

Para Wanke (2001), o ECR tem como objetivo a coordenação de trocas de informações entre indústrias e empresas varejistas com o intuito de gerar um fluxo de produtos e estoques que estejam em sintonia com as informações de vendas, que são obtidas em tempo real nos PDV.

$\mathrm{Na}$ Figura 2, é possível identificar um esquema envolvendo as quatro estratégias e questões relacionadas a práticas, técnicas e ferramentas do ECR.

Figura 2 - ECR e a relação com as estratégias

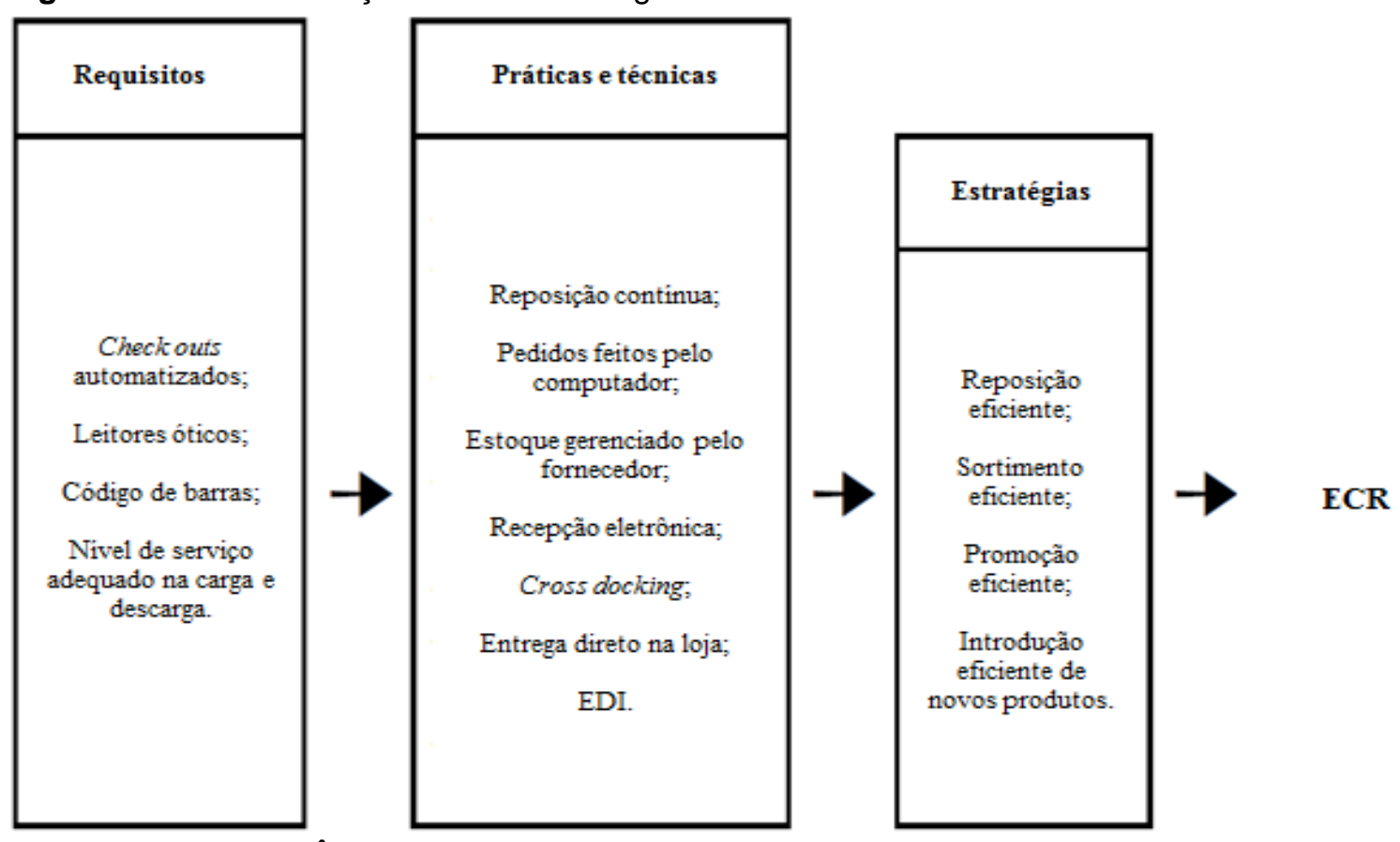

Fonte: Adaptado de Ângelo e Siqueira (2000, p. 96)

Segundo Pires (2009), o ECR está vinculado à lógica do Quick Response (Resposta Rápida), sendo que a demanda ou reposição de um item é puxada pelo ponto de consumo. O ECR tem como principal marca a gestão por categorias de Revista Produção Online. Florianópolis, SC, v.17, n. 2, p. 567-591, 2017. 
produtos (viabilizada pelo CR), administrando, assim, a lucratividade e o estoque e buscando otimizar o mix de produtos e suas respectivas promoções. O autor aponta também que o ECR ajuda a diminuir o trade-off entre o nível de serviço prestado aos clientes e os custos logísticos, proporcionando benefícios tanto para fornecedores (melhora na gestão da demanda, entregas precisas, redução de custos e de burocracia, redução de estoque e diminuição nas perdas), como para varejistas (aumento da exposição e opções de produtos, redução de preços e diminuição do número de perdas com produtos vencidos).

O ECR Brasil (2001) afirma que a implantação do ECR pode trazer também outras vantagens, como pesquisas mais sofisticadas sobre os hábitos de consumo, integração do gerenciamento por categorias de produtos e, ainda, informações sobre a fidelidade dos consumidores.

Concomitante à visão de Ângelo e Siqueira (2000), Wanke (2001) afirma que a prática proporciona melhorias nos seguintes pontos que envolvem uma cadeia de suprimentos: (a) determinação do mix de produtos, mais precisa nos PDV; (b) reabastecimento do mix de produtos em quantidades corretas e nos momentos em que a demanda ocorre; (c) promoções; (d) redução das chances de fracasso no lançamento de novos produtos.

Wanke (2001) ainda destaca a importância do EDI para uma SC pautada pelo ECR, pois a tecnologia permite a transmissão eletrônica em tempo real para os fabricantes do consumo dos PDV na medida de sua ocorrência e a transmissão eletrônica de demanda futura projetada desses pontos de venda.

A adoção do ECR tem impactos positivos sobre o desempenho econômico de fornecedores bem como no desenvolvimento de capacidades, mas em diversos casos cria uma percepção de desigualdade negativa por parte do fornecedor, impulsionada pela maior capacidade do varejo e a falta de confiança que ainda existe entre fornecedor e cliente (CORSTEN e KUMAR, 2005). Por esse motivo, a prática deve ser vista como uma postura de negócios, na qual as empresas da SC se dispõem a compartilhar problemas, dificuldades e informações e implementar as melhores soluções dentro de seu contexto operacional e estratégico, resultando em uma efetiva gestão colaborativa. 


\subsection{Collaborative Planning, Forecasting and Replenishment}

Segundo Pires (2009), não existe um consenso na academia sobre o início da prática de CPFR (Planejamento, Previsão e Reposição/Reabastecimento Colaborativos), visto que alguns autores indicam seu surgimento em 1995, com a aplicação dessa prática pela rede varejista Wal-Mart, enquanto outros autores defendem que o marco do CPFR foi estabelecido em 1998 pela Voluntary Interindustry Commerce Standard (VICS), que é uma instituição voluntária nos Estados Unidos criada com o objetivo de auxiliar empresas no compartilhamento de informações e gerenciamento de seus processos.

Considerada uma evolução do VMI, CR e do ECR, essa prática tem como objetivo básico eliminar as falhas observadas nas ferramentas anteriores. Isso porque o CPFR se preocupa com questões não abordadas nas demais práticas, como a falta de sincronização e integração entre os processos executados nos diversos setores da cadeia, a falta de coordenação entre pontos de venda, processo de compras e planejamento logístico para os varejistas e a união dos diversos métodos de previsão usados na mesma empresa ou SC, trazendo uma nova realidade ao planejamento e aos processos colaborativos entre as empresas (BARRATT e OLIVEIRA, 2001).

Nesse sentido, o CPFR é um modelo para cadeias de suprimentos que auxilia na organização e compartilhamento de informações em conjunto com a finalidade de equilibrar as relações entre os elos por meio do controle de estoque em todas as empresas envolvidas (VIVALDINI, PIRES e SOUZA, 2008).

Todavia, algumas questões antecedem as atividades colaborativas em CPFR, como a adequação de normas comuns de informações e o registro e a sincronização de dados (VOLUNTARY INTERINDUSTRY COMMERCE STANDARDS, 2004). Barratt e Oliveira (2001) também mencionam que outros obstáculos são representados pela falta de uma maior disciplina ao se executar as duas primeiras fases de um projeto CPFR, que são o acordo inicial e o desenvolvimento do plano de negócio. Outros fatores são a definição de níveis de serviço distintos entre os pontos de venda, o conhecimento dos custos e da capacidade total da SC, a reposição ineficiente como resposta às flutuações de demanda, o planejamento 
ineficiente a partir das informações dos pontos de vendas e a obtenção de escalas de produção adequadas.

Somente depois de vencidas essas barreiras iniciais, as empresas da cadeia podem partir para o desenvolvimento das atividades colaborativas de gestão da demanda e abastecimento, estratégia e planejamento, execução, monitoramento e análise viabilizadas pelo CPFR (VOLUNTARY INTERINDUSTRY COMMERCE STANDARDS, 2004). Pires (2009) faz uma interessante compactação, com base em diversos autores, sobre os principais benefícios e as mais importantes barreiras e desafios envolvendo o CPFR, apresentados de maneira sucinta no Quadro 5.

$\mathrm{Na}$ concepção de Vivaldini, Pires e Souza (2008), outras questões que envolvem a implantação dos processos de CPFR são o desenvolvimento de acordos na linha de frente dos processos de negócios, a criação de planos de negócios conjuntos, a criação das previsões de vendas individuais, a identificação de exceções nas previsões de vendas, a colaboração em soluções de itens de exceção, a criação da previsão de pedidos, a identificação de exceções em previsão de pedidos e a geração de pedidos.

Quadro 5 - Benefícios, barreiras e desafios do CPFR

\begin{tabular}{|c|c|c|}
\hline Benefícios & Barreiras & Desafios \\
\hline $\begin{array}{l}\text { - Ciclos de atendimento mais } \\
\text { previsíveis. } \\
\text { - Carregamentos menores. } \\
\text { - Maior atualização, agilidade e } \\
\text { nível de precisão no fluxo de } \\
\text { informações. } \\
\text { - Maior formatação da } \\
\text { informação para facilitar seu } \\
\text { uso. } \\
\text { - Facilita práticas de } \\
\text { postponement na SC e de } \\
\text { assemble to order na empresa. } \\
\text { - Aumento no nível de serviço } \\
\text { ao cliente. } \\
\text { - Equilíbrio entre faltas e } \\
\text { excessos de estoques. } \\
\text { - Conectividade e integração } \\
\text { na SC. } \\
\text { - Redução dos custos de } \\
\text { estoque e custos totais. }\end{array}$ & $\begin{array}{l}\text { - Provisão de uma tecnologia } \\
\text { adequada de hardware e } \\
\text { software para suporte } \\
\text { adequado do processo. } \\
\text { - Dificuldades de coordenação } \\
\text { de troca de informações em } \\
\text { tempo real. } \\
\text { - Investimento em tempo e } \\
\text { pessoas para preparar o } \\
\text { processo. } \\
\text { - Manutenção de esforços na } \\
\text { SC. } \\
\text { - Falta de escala para o projeto } \\
\text { piloto. } \\
\text { - Mudanças culturais voltadas } \\
\text { para a colaboração. }\end{array}$ & $\begin{array}{l}\text { - Criação de um guia prático } \\
\text { para orientar a implementação } \\
\text { do CPFR. } \\
\text { - Definir quais informações } \\
\text { devem ser compartilhadas. } \\
\text { - Definir quais funções e } \\
\text { processos devem ser } \\
\text { envolvidos. }\end{array}$ \\
\hline
\end{tabular}

Fonte: Adaptado de Pires (2009, p. 170)

\section{MÉTODO}

Revista Produção Online. Florianópolis, SC, v.17, n. 2, p. 567-591, 2017. 
A pesquisa realizada caracteriza-se como um estudo bibliométrico, com método quantitativo e de natureza descritiva. Foi utilizada como base para a coleta dos artigos sobre o tema práticas e iniciativas colaborativas em cadeias de suprimentos a base internacional Scopus. A escolha dessa base de dados se deu por conta de sua relevância para o meio acadêmico e pela extensão de sua abrangência. A Scopus hoje conta com a cobertura de mais de 21 mil periódicos de mais de 5 mil editoras internacionais (ELSEVIER, 2015). Para Collis e Hussey (2012), a pesquisa descritiva é caracterizada pelo comportamento dos fenômenos, para obter informações sobre características de um determinado problema.

Para o tratamento dos dados obtidos, foram utilizados os indicadores encontrados na própria base de dados e a ferramenta Microsoft Excel, empregada em tabulações, formatações e criação das tabelas e figuras para posterior análise. Os dados coletados são apresentados na próxima seção.

\subsection{Amostragem e coleta de dados}

A coleta de dados ocorreu no mês de junho de 2015 a partir da delimitação de um período de dez anos (2005-2014), sendo pesquisados os seguintes termoschave: "Electronic Data Interchange", "Efficient Customer Response", "Continuous Replenishment", "Vendor Managed Inventory", e "Collaborative Planning, Forecasting and Replenishment".

A pesquisa foi realizada nos títulos, subtítulos e palavras-chave dos artigos na subárea de "business, management and accounting". A procura desses termoschave retornou 331 artigos da base Scopus, sendo esse o tamanho da amostragem do presente trabalho.

\subsection{Categorias para análise dos artigos}

A partir das proposições elencadas para a pesquisa, foram delimitadas as categorias de análise dos dados, conforme demonstra o Quadro 6.

Quadro 6 - Categorias de análise da pesquisa

$$
\text { Proposição }
$$

Categoria de análise

Revista Produção Online. Florianópolis, SC, v.17, n. 2, p. 567-591, 2017. 


\begin{tabular}{|l|l|}
\hline $\begin{array}{l}\text { (a) há concentração de publicações em } \\
\text { poucos periódicos }\end{array}$ & (a) número de publicações por periódicos \\
\hline $\begin{array}{l}\text { (b) existe predominância de publicações em } \\
\text { alguns países }\end{array}$ & (b) número de publicações por países \\
\hline $\begin{array}{l}\text { (c) há claramente alguns autores com maior } \\
\text { número de publicações sobre os temas }\end{array}$ & $\begin{array}{l}\text { (c) autores com maior número de produção } \\
\text { de artigos }\end{array}$ \\
\hline $\begin{array}{l}\text { (d) existem práticas e iniciativas que se } \\
\text { destacam em relação a quantidade de } \\
\text { pesquisas }\end{array}$ & $\begin{array}{l}\text { (d) práticas e iniciativas com maior número de } \\
\text { produção de artigos }\end{array}$ \\
\hline $\begin{array}{l}\text { (e) as publicações sobre os temas cresceram } \\
\text { conforme o passar dos anos }\end{array}$ & (e) evolução das publicações sobre os temas \\
\hline
\end{tabular}

Fonte: Elaborado pelos autores

\section{RESULTADOS DA PESQUISA}

A seguir são apresentados os resultados obtidos pelo levantamento bibliométrico e uma breve discussão sobre cada categoria de análise, levando em conta as especificidades de cada categoria e sua relação com as proposições de pesquisa.

\subsection{Publicações por periódicos}

A Tabela 1 apresenta os periódicos com maior número de publicações para os termos utilizados na pesquisa. É importante ressaltar que os dados são apresentados sem uma ordem específica, o que ocorre também nas demais categorias de análise.

Tabela 1 - Número de publicações por periódicos

\begin{tabular}{lcccccc}
\hline \multicolumn{1}{c}{ Países } & EDI & ECR & CR & VMI & CPFR & TOTAL \\
\hline Demais periódicos* & 120 & 15 & 5 & 64 & 25 & 229 \\
International Journal of Production Economics & - & - & - & 18 & 2 & 20 \\
International Journal of Production Research & - & - & - & 17 & - & 17 \\
Information and Management & 9 & - & - & - & - & 9 \\
International Journal of Logistics Systems and & - & - & - & 9 & - & 9 \\
Management & - & & & &
\end{tabular}

Revista Produção Online. Florianópolis, SC, v.17, n. 2, p. 567-591, 2017. 
Supply Chain Management

Transportation Research Part E Logistics and

Transportation Review

Journal of Management Information Systems

Decision Support Systems

Industrial Management and Data System

International Journal of Physical Distribution and

Logistics Management

Decision Support Systems

Journal of Manufacturing Technology Management

TOTAL

* Periódicos que aparecem com frequência menor de cinco vezes para cada um dos termos de pesquisa utilizados.

Fonte: Elaborado pelos autores com base nos dados da pesquisa

Com base nos dados coletados, pode-se afirmar que os periódicos com maior número de publicações, no geral, foram o International Journal of Production Economics (20) e o International Journal of Production Research (17), com um total de 37 publicações e uma representatividade de $11,18 \%$ do total da amostra. A linha "demais periódicos", na Tabela 1, representa os periódicos que tiveram até quatro artigos publicados sobre os temas.

\subsection{Publicações por países}

A seguir são elencados os países com maior número de publicações para os termos de pesquisa listados. A Tabela 2 apresenta a separação das cinco práticas e iniciativas escolhidas para a pesquisa e suas representações nos países através de suas quantidades de artigos.

Tabela 2 - Número de publicações por países

\begin{tabular}{lcccccc}
\hline \multicolumn{1}{r}{ Países } & EDI & ECR & CR & VMI & CPFR & TOTAL \\
\hline Estados Unidos & 64 & 6 & 3 & 38 & 11 & 122 \\
Demais países & 31 & - & - & 8 & 2 & 41 \\
Reino Unido & 13 & 5 & - & 7 & 3 & 28 \\
China & 8 & 1 & 1 & 15 & 3 & 28 \\
Índia & - & - & 1 & 18 & 4 & 23 \\
Hong Kong & 9 & - & - & 6 & 2 & 17 \\
Taiwan & - & - & - & 10 & 3 & 13 \\
Canadá & 5 & - & - & 7 & - & 12 \\
Coreia do Sul & 10 & - & - & - & - & 10
\end{tabular}

Revista Produção Online. Florianópolis, SC, v.17, n. 2, p. 567-591, 2017. 


\begin{tabular}{lllllll} 
Itália & - & - & - & 7 & - & 7 \\
Austrália & 6 & - & - & - & - & 6 \\
Singapura & 5 & - & - & - & - & 5 \\
Finlândia & - & - & - & 5 & - & 5 \\
Irã & - & - & - & 5 & - & 5 \\
Turquia & - & - & - & 4 & - & 4 \\
Brasil & - & 1 & - & - & 2 & 3 \\
Alemanha & - & 2 & - & - & - & 2 \\
\hline \multicolumn{7}{l}{ TOTAL } \\
* Países que aparecem com frequência inferior a quatro vezes para cada um dos termos de \\
pesquisa utilizados. \\
Fonte: Elaborado pelos autores com base nos dados da pesquisa
\end{tabular}

Nota-se nos dados uma grande concentração de publicações nos Estados Unidos (122), com uma representatividade de quase metade da amostra analisada. Destacam-se ainda o Reino Unido e China, com 28 publicações cada, e a Índia, com 23 publicações.

\subsection{Autores com maior número de artigos publicados}

Nesta etapa, são apresentados os resultados referentes aos autores com maior número de artigos publicados.

Para o termo "Electronic Data Interchange" foram encontrados 18 autores que, apesar de aparecem com apenas dois artigos cada, tiveram o maior número de publicações: Azedeh, A.; Kauffman, R. J.; Hill, C. A.; Hanseth, O.; Dresner, M.; Howard, M.; Lee, S.; Kim, K. K.; Nicolaou, A. I.; Pan, G.; Power, D.; Ramdeen, C. D.; Santos, J.; Scudder, G. D.; Sundarraj, R. P.; Umanath, N. S.; Yen, D. C. e Zhu, K. Os demais autores aparecem com apenas uma publicação cada.

Nos termos "Efficient Customer Response" e "Continuous Replenishment" não foram encontrados autores com um número maior do que uma publicação cada.

Para o termo "Vendor Managed Inventory", os autores que mais publicaram foram: Dresner, M., com 6 artigos; Borade, A. B., com 5 artigos; e Holmstrom, J.; Bansod, S. V.; Yao, Y.; Niaki, S. T. A, com 4 artigos cada. Demais autores publicaram três ou menos artigos.

Já no termo "Collaborative Planning Forecasting and Replenishment" aparecem com duas publicações os autores: Kohli, R.; Cederlund, J.; Sherer, S. A.;

Revista Produção Online. Florianópolis, SC, v.17, n. 2, p. 567-591, 2017. 
Yao, Y.. Outros autores possuem apenas uma publicação cada sobre o termo analisado.

Destacam-se aqui os autores Dresner, M., com duas publicações para o termo "Electronic Data Interchange" e seis publicações com o termo "Vendor Managed Inventory", e o autor Yao, Y., com quatro artigos sobre "Vendor Managed Inventory" e mais dois artigos com o termo "Collaborative Planning Forecasting and Replenishment".

\subsection{Práticas e iniciativas com maior produção e evolução das publicações}

A Tabela 3 propicia uma análise sobre os dados coletados em relação às práticas e iniciativas mais pesquisadas nos últimos dez anos, assim como a evolução das publicações sobre os temas nesse período.

Os dados coletados permitem afirmar que os termos mais representativos encontrados na pesquisa foram "Electronic Data Interchange", com 151 artigos, e "Vendor Managed Inventory", com 130 artigos, compondo, juntos, quase $85 \%$ da amostra total. Assim, tende-se a concluir que esses dois temas são particularmente importantes no âmbito da gestão colaborativa em cadeias de suprimentos, muito em face de suas características apresentadas na revisão bibliográfica.

Já os termos "Collaborative Planning Forecasting and Replenishment", "Efficient Customer Response" e "Continuous Replenishment" foram representados por, respectivamente, 30, 15 e 5 artigos somente. Nos três casos, nota-se que a menor quantidade de trabalhos pode se dever às dificuldades de implementação das práticas relacionadas, conforme notado por Pires (2009), ou seja, é possível que poucas pesquisas estejam sendo conduzidas sobre os temas porque poucas empresas têm conseguido viabilizar a utilização dessas práticas e iniciativas em suas cadeias de suprimentos.

Tabela 3 - Práticas e iniciativas com maior produção e evolução das publicações

\begin{tabular}{ccccccc}
\hline $\begin{array}{c}\text { Ano/Iniciativa ou } \\
\text { Prática Colaborativa }\end{array}$ & EDI & ECR & CR & VMI & CPFR & TOTAL \\
\hline 2005 & 22 & 2 & 0 & 4 & 1 & $\mathbf{2 9}$ \\
2006 & 18 & 3 & 0 & 11 & 2 & $\mathbf{3 4}$ \\
2007 & 19 & 2 & 0 & 14 & 5 & $\mathbf{4 0}$
\end{tabular}

Revista Produção Online. Florianópolis, SC, v.17, n. 2, p. 567-591, 2017. 


\begin{tabular}{ccccccc}
2008 & 17 & 2 & 1 & 16 & 3 & $\mathbf{3 9}$ \\
2009 & 15 & 2 & 0 & 12 & 3 & $\mathbf{3 2}$ \\
2010 & 16 & 2 & 1 & 14 & 3 & $\mathbf{3 6}$ \\
2011 & 12 & 0 & 0 & 9 & 2 & $\mathbf{2 3}$ \\
2012 & 8 & 0 & 1 & 16 & 3 & $\mathbf{2 8}$ \\
2013 & 14 & 0 & 1 & 13 & 3 & $\mathbf{3 1}$ \\
2014 & 10 & 2 & 1 & 21 & 5 & $\mathbf{3 9}$ \\
\hline TOTAL & $\mathbf{1 5 1}$ & $\mathbf{1 5}$ & $\mathbf{5}$ & $\mathbf{1 3 0}$ & $\mathbf{3 0}$ & $\mathbf{3 3 1}$ \\
\hline
\end{tabular}

Fonte: Elaborado pelos autores com base nos dados da pesquisa

Em relação à categoria de análise denominada evolução das publicações sobre os temas, nota-se, na Tabela 4, que não foi possível identificar uma predominância ou concentração de publicações em determinado ano ou período, havendo um equilíbrio na quantidade de publicações distribuídas nos dez anos que abrangeram a pesquisa. Todavia, é marcante a queda de trabalhos sobre EDI e a evolução de pesquisas sobre VMI. Assim, é possível supor que práticas colaborativas, como o VMl, ainda estão bastante presentes nas organizações, embora possam estar sendo implementadas através de outros recursos tecnológicos, como o XML (eXtensible Markup Language) em detrimento do EDI.

\section{CONSIDERAÇÕES FINAIS}

O objetivo principal desta pesquisa foi analisar a produção acadêmica internacional a partir de artigos indexados no Scopus sobre as principais práticas e iniciativas colaborativas em cadeias de suprimentos. Desse modo, entende-se que foi possível atingir esse propósito e responder à pergunta-problema com a apresentação dos resultados e discussões de elementos como os periódicos e países onde foram publicados os artigos, a quantidade de artigos publicados por ano, os autores que mais publicaram, as práticas e iniciativas que mais se destacam e a evolução das publicações sobre os temas.

No que diz respeito às proposições de pesquisa, algumas delas puderam ser confirmadas, enquanto outras foram refutadas. A proposição "a" (há concentração de publicações em poucos periódicos?) não pôde ser confirmada, pois periódicos que aparecem com frequência igual ou inferior a quatro totalizaram 229 das 331 
ocorrências, ou seja, $69 \%$ da amostra. Assim, embora periódicos como 0 International Journal of Production Economics e o International Journal of Production Research se destaquem na pesquisa, a grande maioria de artigos sobre os temas está espalhada entre diversas publicações internacionais.

Já quanto à proposição "b" (existe predominância de publicações em alguns países?), ela pode ser confirmada pois os Estados Unidos detêm 36,86\% do total das publicações. Desse modo, é possível afirmar que a maioria das pesquisas sobre as práticas e iniciativas de gestão colaborativa em cadeias de suprimentos vem sendo conduzida na América do Norte.

A proposição "c" (há claramente alguns autores com maior número de publicações sobre os temas?), foi refutada devido a um grande número de autores com apenas uma publicação e à baixa proporção de autores em relação aos temas. Mesmo no caso de Dresner, M., que conta com duas publicações sobre EDI e seis publicações sobre VMI, e Yao, Y., com quatro artigos sobre VMI e dois sobre CPFR, a representatividade pode ser considerada baixa em relação à amostra, representando apenas $2,41 \%$ e $1,81 \%$ do total, respectivamente. Para que um autor fosse considerado com, claramente, um maior número de publicações sobre os temas, esperava-se ao menos uma proporção de 5\% dos trabalhos.

Quanto à proposição "d" (existem práticas e iniciativas que se destacam em relação à quantidade de pesquisas?), foi possível confirmá-la ao serem encontrados 151 artigos desenvolvidos sobre EDI e 130 artigos sobre VMI. Esses números representam $45,61 \%$ e $39,27 \%$ da amostra total, respectivamente, o que indica clara importância dessas duas práticas para a gestão colaborativa em cadeias de suprimentos e um grande foco da academia sobre os desafios e benefícios dessas ferramentas.

Por fim, a proposição "e" (as publicações sobre os temas cresceram conforme o passar dos anos?) foi refutada ao identificar-se que houve uma constante no número de publicações ao longo dos dez anos analisados. No ano de 2007, encontrou-se um total de 40 artigos (maior índice) e em 2011 um total de 23 artigos (menor índice), auferindo-se uma média de 33 artigos publicados por ano. De todo modo, a análise desses dados permitiu a compreensão sobre a diminuição de trabalhos sobre EDI ao longo dos anos, enquanto a pesquisa sobre VMI mostra uma 
notável linha ascendente. Esse fato pode estar ocorrendo devido à substituição da tecnologia EDI por outras formas de transmissões de dados, como o XML, e à constante implementação de estoques gerenciados pelo fornecedor nas organizações.

É certo que esta pesquisa é limitada pelo fato de analisar um horizonte de tempo de dez anos, o que pode não abranger a totalidade das pesquisas desenvolvidas sobre os termos pesquisados, podendo haver outras práticas e iniciativas colaborativas em cadeias de suprimentos, o que sugere a possibilidade de análises complementares. Nesse sentido, tanto um comparativo entre as demais práticas e iniciativas colaborativas como a ampliação da amostra poderiam proporcionar generalizações mais precisas sobre o tema geral e seus correlatos aqui estudados. Todavia, ao identificar o panorama dos últimos dez anos sobre as pesquisas relacionadas às principais práticas e iniciativas colaborativas em cadeias de suprimentos, entende-se que foi possível demonstrar a importância de cada uma delas dentro dos cenários acadêmico e corporativo.

\section{REFERÊNCIAS}

ALMEIDA, M. M. K de; MARINS, F. A. S.; TRAMARICO, C. L. O gerenciamento do relacionamento com fornecedores na integração da cadeia de suprimentos: um estudo de caso na indústria eletromecânica. Revista Gestão Industrial, Ponta Grossa, v. 8, n. 3, p. 184-205, 2012. doi: 10.3895/S1808-04482012000300009

ÂNGELO, C. F. de; SIQUEIRA, J. P. L. de. Avaliação das condições logísticas para a adoção do ECR nos supermercados brasileiros. Revista de Administração

Contemporânea, Rio de Janeiro, v. 4, n. 3, p. 89-106, 2000. doi: 10.1590/S1415$\underline{65552000000300006}$

ARUNACHALAM, S. Science on the periphery: bridging the information divide. In: MOED, $H$. F. et al. (Ed.). Handbook of quantitative science and technology research. Netherlands: Kluwer, 2004. p. 163-183.

BARRATT, M.; OLIVEIRA, A. Exploring the experiences of collaborative planning initiatives. International Journal of Physical Distribution \& Logistics, v. 31, n. 4, p. 266-289, 2001. doi: $\underline{10.1108 / 09600030110394932}$

BLATHERWICK, A. Vendor-managed inventory: fashion fad or important supply chain strategy? Supply Chain Management: An International Journal, Bingley, v. 3, n. 1, p. 10-11, 1998. doi: $\underline{10.1108 / 13598549810200825}$ 
CACHON, G.; FISHER, M. Campbell soup's continuous replenishment program: evaluation and enhanced inventory decision rules. Production and Operations Management, Hobokoen, v. 6, n. 3, p. 266-276, 1997. doi: 10.1111/j.1937-5956.1996.tb00430.x

CAMARGO JUNIOR, J. B. Um modelo de utilização de computação em nuvens para empresas atuando em cadeias de suprimentos operando no Brasil. 2015. $170 \mathrm{f}$. Tese (Doutorado em Administração) - Faculdade de Gestão e Negócios, Universidade Metodista de Piracicaba, Piracicaba, 2015.

CHEN, L. T. Dynamic supply coordination under consignment and vendor managed inventory in retailer-centric BSB electronic markets. Industrial Marketing Management, Amsterdam, v. 42, n. 4, p. 518-531, 2013. doi: 10.1016/j.indmarman.2013.03.004

CHRISTOPHER, M.; TOWILL, D. An integrated model for the design of agile supply chains, International Journal of Physical Distribution \& Logistics Management, Bingley, v. 31, n. 4, p. 235-246, 2001. doi: 10.1108/09600030110394914

COLLIS, J.; HUSSEY, R. Pesquisa em administração: um guia prático para alunos de graduação e pós-graduação. $3^{\text {a }}$ ed. São Paulo: Atlas, 2012.

CORSTEN, D.; KUMAR, N. Do suppliers benefit from collaborative relationships with large retailer? An empirical investigation of efficient consumer response adoption. Journal of Marketing, Chicago, v. 69, n. 3, p. 80-94, 2005. doi: $10.1509 /$ jmkg.69.3.80.66360

COX, A. The art of possible: Relationship management in power regimes and supply chains. Supply Chain Management: An International Journal, Bingley, v. 9, n. 5, p. 346-356, 2004.

ECR BRASIL. Os novos desafios do gerenciamento por categorias. Boletim da Associação ECR Brasil sobre eficiência empresarial, São Paulo, ano 1, n. 2, nov. 2001. Disponível em: <https://goo.gl/VTssqL>. Acesso em: 3 maio 2017.

ELSEVIER. Scopus content. Elsevier, Amsterdam, 2015. Disponível em: < http://www.elsevier.com/solutions/scopus/content>. Acesso em: 8 jul. 2015.

FERREIRA, K. A.; ALVES, M. R. P. A. Logística e troca eletrônica de informação em empresas automobilísticas e alimentícias. Produção, São Paulo, v. 15, n. 3, p. 434-447, 2005. doi: 10.1590/S0103-65132005000300012

GOVINDAN, K. Vendor-managed inventory: a review based on dimensions. International Journal of Production Research, London, v. 51, n. 13, p. 3808-3835, 2013. doi: $\underline{10.1080 / 00207543.2012 .751511}$

HARIGA, M., et al. A vendor managed inventory model under contractual storage agreement. Computers \& Operations Research, Amsterdam, v. 40, n. 8, p. 21382144 , 2013. doi: $\underline{10.1016 / \text { j.cor.2013.03.005 }}$

HOFFMAN, J. M.; MEHRA, S. Efficient consumer response as a supply chain strategy for grocery businesses. International Journal of Service Industry Management, Bingley, $v$. 11 , n. 4, p. 365-373, 2000. doi: $10.1108 / 09564230010355386$

KNOFF, L. L. et al. Mapeamento de publicações científicas sobre estratégia de manufatura: uma abordagem baseada em processos. Revista Produção Online, Florianópolis, v. 14, n. 1, p. 403-429, 2014. Doi: 10.14488/1676-1901.v14i2.1043

Revista Produção Online. Florianópolis, SC, v.17, n. 2, p. 567-591, 2017. 
KURNIA, S.; SWATMAN, P. M. C.; SCHAUDER, D. Efficient consumer response: a preliminary comparison of US and European experiences. In: INTERNATIONAL CONFERENCE ON ELECTRONIC COMMERCE, 11., 1998, Bled. Proceedings... New York: Association for Computing Machinery, 1998. p. 126-143.

LAMBERT, D. M.; COOPER, M. C.; PAGH, J. D. Supply chain management: implementation issues and research opportunities. The International Journal of Logistics Management, Bingley, v. 9, n. 2 , p. 1-20, 1998. doi: $\underline{10.1108 / 09574099810805807}$

LEE, J. Y.; REN, L. Vendor-managed inventory in a global environment with Exchange rate uncertainty. Internationl Journal of Production Economics, Amsterdam, v. 130, n. 2, p. 169-174, 2011. doi: 10.1016/j.jipe.2010.12.006

MACHUCA, J. A. D.; BARAJAS, R. P. The impact of electronic data interchange on reducing bullwhip effect and supply chain inventory costs. Transportation Research Part E: Logistics and Transportation Review, Amsterdam, v. 40, n. 3, p. 209-228, 2004. doi:

10.1016/j.tre.2003.08.001

MARTENS, B. J.; DOOLEY, F. J. Food and grocery supply chain: a reappraisal of ECR performance. International Journal of Physical Distribution \& Logistics Management, Bingley, v. 40, n. 7, p. 534-549, 2010. doi: $\underline{10.1108 / 09600031011071993}$

PEREIRA, S. E. H.; PIRES, S. I. R.; SILVA, E. M. da. Análise da prática do VMI no abastecimento de oxigênio e de materiais de consumo de um hospital público. In: SIMPÓSIO DE ADMINISTRAÇÃO DA PRODUÇÃO, LOGÍSTICA E OPERAÇÕES INTERNACIONAIS - SIMPOI, 16., 2013, São Paulo. Anais... São Paulo: FGV-EASP, 2013. p. 1-16.

PIRES, S. R. I. Gestão da cadeia de suprimentos (supply chain management): conceitos, estratégias, práticas e casos. 2. ed. São Paulo: Atlas, 2009.

PORTAL EDI. O que é EDI? Disponível em: <http://www.logicainfo.com.br/servicos/5/edi--eletronic-datainterchange/>. Acesso em: 25 out. 2013.

RESENDE, P. T. V. de; MENDONÇA, G. D. CPFR: uma técnica colaborativa aplicada ao contexto corporativo brasileiro. In: ENCONTRO DA ASSOCIAÇÃO NACIONAL DE PÓSGRADUAÇÃO E PESQUISA EM ADMINISTRAÇÃO - ANPAD, 30., 2006, Salvador.

Anais..., Salvador: ANPAD, 2006

RIZZARDO JUNIOR, M. A. G.; PORTO, R. M. CPFR - Collaborative Planning Forecasting and Replenishment: indústria e varejo aprimorando a gestão de estoques no setor de alimentos. In: SIMPÓSIO DE ADMINISTRAÇÃO DA PRODUÇÃO, LOGÍSTICA E OPERAÇÕES INTERNACIONAIS - SIMPOI, 11., 2008, São Paulo. Anais... São Paulo: FGV-EASP, 2008. p. 1-14

RODRIGUES, M. C. N.; PROVIDELLO, M. N. de L.; BAGNO, R. B. A influência da engenharia de produção nos serviços de atendimento à saúde: estudo bibliométrico focado em técnicos operacionais. Revista Produção Online, Florianópolis, v. 16, n.1, p. 242-262, 2016. doi: $\underline{10.14488 / 1676-1901 . v 16 i l .2088}$ 
RODRIGUES, D. M.; SELLITTO, M. A. Práticas logísticas colaborativas: o caso de uma cadeia de suprimentos da indústria automobilística. Revista de Administração, São Paulo, v. 43 , n. 1, p. $97-111,2008$.

SAAB JUNIOR, J. Y.; CORRÊA, H. L. Cadeia de abastecimento: gestão do estoque pelo distribuidor. Revista de Administração de Empresas, v. 48, n. 1, p. 48-62, 2008.

TAN, K. C.; KANNAN, V. R.; HSU, C. C. Supply chain information and relational alignments: mediators of EDI on firm performance. International Journal of Physical Distribution \& Logistics Management, Bingley, v. 40, n. 5, p. 377-394, 2010. doi: $10.1108 / 09600031011052831$

VOLUNTARY INTERINDUSTRY COMMERCE STANDARDS. Collaborative Planning, Forecasting and Replenishment (CPFR). 2004. Disponível em:

<http://www.vics.org/docs/committees/cpfr/CPFR_Overview_US-A4.pdf>. Acesso em: 18 set. 2013.

VITORINO FILHO, V. A. et al. Gestão colaborativa em cadeias de suprimentos: um estudo bibliométrico. Gestão \& Regionalidade, v. 32, n. 96, p. 111-134, 2016. doi:

10.13037/gr.vol32n96.3527

VITORINO FILHO, V. A. et al. A produção acadêmica internacional em gestão de operações: um estudo bibliométrico. Revista Produção Online, Florianópolis, v. 15, n. 1, p. 21-49, 2015. doi: $10.14488 / 1676-1901 . v 15 i l .1574$

VIVALDINI, M. O prestador de serviços logísticos como agente integrador em cadeias de suprimentos: uma proposta de sistematização conceitual. 2007. 167 f. Tese (Doutorado em Engenharia de Produção) - Universidade Metodista de Piracicaba, Santa Bárbara do Oeste, 2007.

VIVALDINI, M.; PIRES, S. R. I.; SOUZA, F. B. de. Fatores não tecnológicos envolvidos na implementação e gestão de um CPFR. In: SIMPÓSIO DE ADMINISTRAÇÃO DA PRODUÇÃO, LOGÍSTICA E OPERAÇÕES INTERNACIONAIS - SIMPOI, 11., 2008, São Paulo. Anais... São Paulo: FGV-EASP, 2008. p. 1-14.

VIVALDINI, M.; PIRES, S. R. I.; SOUZA, F. B. de. Importância dos fatores não tecnológicos na Implementação do CPFR. Revista de Administração Contemporânea, Rio de Janeiro, v. 14, n. 2, p. 289-309, 2010.

VIVALDINI, M.; SOUZA, F. B. de. O relacionamento colaborativo na cadeia de suprimentos do McDonald's. In: SIMPÓSIO DE ENGENHARIA DE PRODUÇÃO - SIMPEP, 13., 2006, Bauru. Anais... Bauru: Unesp, 2006.

WANKE, P. Efficient consumer response (ECR): a logística de suprimentos just-in-time aplicada ao varejo. 2001. Disponível em: <https://goo.gl/iDVqvG>. Acesso em: 18 set., 2013.

WANKE, P. Uma revisão dos programas de resposta rápida: ECR, CRP, VMI, CPFR, JIT II. Especialistas em Logística e Supply Chain, Rio de Janeiro, 10 jun. 2004. Disponível em: $<$ https://goo.gl/f5n6cc>. Acesso em: 18 set., 2013. 
YU, Y.; WANG, Z.; LIANG, L. A vendor managed inventory supply chain with deteriorating raw materials and products. International Journal of Production Economics, Amsterdam, v. 136, n. 2, p. 266-274, 2012.

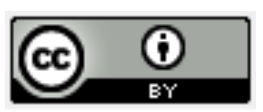

Artigo recebido em 18/05/2016 e aceito para publicação em 05/05/2017

DOI: http://dx.doi.org/10.14488/1676-1901.v17i2.2462

Revista Produção Online. Florianópolis, SC, v.17, n. 2, p. 567-591, 2017. 